\title{
Communications
}

\section{Collège de Médecine de Premier Recours CMPR}

Prix CMPR de recherche en médecine de premier recours 2012

Spécialité: Médecine de premier recours Critères des travaux: Travaux scientifiques achevés en Suisse ou par des auteurs suisses habitant à soumis l'étranger, qui apportent une contribution importante aux soins de base offerts par le médecin de premier recours, notamment: à la qualité du traitement et de la prise en charge des patients de premier recours, à la pratique médicale des médecins de premier recours, (thématique valable, pertinente et réalisable en pratique), à la recherche sur le cabinet du médecin de premier recours: fondements, conditions de travail, gestion et préservation des ressources.

Prix: 30000 francs offert par la maison Mepha Pharma SA. Le prix sera accordé à un seul lauréat pour son travail scientifique ou alors il sera réparti sur plusieurs auteurs (sans possibilité de recours devant les tribunaux).

Participant(e)s: Auteurs en Suisse ou personnes suisses habitant à l'étranger ayant achevé un travail scientifique majeur sur la médecine de premier recours dans les trois dernières années.

Délai de soumission: 1 décembre 2011 (attention: cette année ce délai est plus rapide).

Remise du prix: Le 21 juin 2012 lors du Congrès de formation continue du CMPR Lucerne (21 et 22 juin 2012), et le 30 août 2012 à Lausanne (Journée de formation CMPR, en commun avec Swiss Family Docs Conference, 30 et 31 août 2012).

Comité Jury: indépendant, nommé par le conseil de fondation du CMPR.

Détail des conditions de participation: dossier à fournir sous forme électronique ou de CD (pas de disquettes) et sous forme papier: formulaire d'inscription (www.kollegium.ch/ rd/f.html), curriculum vitae de l'auteur principal, manuscrit, brève lettre d'accompagnement «Importance de ce travail pour la médecine de premier recours». Nous ne prendrons pas en considération les travaux ayant déjà été soumis une fois et il n'y aura pas d'échange de courrier au sujet de l'attribution des prix.

Lieu de contact: Collège de médecine de premier recours CMPR, Secrétariat de la Recherche en médecine de premier recours, Landhausweg, 26, 3007 Berne, foham@ kollegium.ch; www.kollegium.ch/rd/f.html

\section{Société Suisse du Psoriasis et du Vitiligo (SSPV)}

Nouvelle brochure de conseil en matière de psoriasis

Le thème du psoriasis est traité en détail dans la brochure de conseil publiée par la Société Suisse du Psoriasis et du Vitiligo (SSPV).

La SSPV est une société d'entraide pour patients souffrant de psoriasis et de vitiligo. Dans la brochure, on décrit la nature du psoriasis, les formes qu'il peut prendre ainsi que les facteurs qui déclenchent cette maladie de la peau. Les différentes thérapies, classiques et modernes, médicales et alternatives, sont mentionnées, aussi bien que les soins quotidiens de la peau. Des sujets particuliers, comme la dépression, l'alimentation, l'alcool, le tabac, la sexualité, la grossesse ou le psoriasis chez les enfants, complètent le tableau d'ensemble des informations. Le conseiller est enrichi par une liste de groupes régionaux de la SSPV et d'autres organisations de patients. Des conseils d'ordre général et des références bibliographiques parachèvent le tout.

La brochure peut être obtenue auprès du secrétariat de la SSPV, Scheibenstrasse 20, 3014 Berne, tél. 03135990 99, info@spvg.ch

\section{evidentia}

evidentia.ch - Informationsplattform von Ärzten für Ärzte

Das schweizerische Evidenzportal für Diagnostik und Therapie www.evidentia.ch stellt kostenlos ständig aktualisierte, evidenzbasierte Informationen für Schweizer Fachärzte in Klinik, Praxis und Ausbildung zur Verfügung. Slide Kits zum Download geben einen umfassenden Überblick über eine Indikation (z. B. Diagnose und Therapie des Hirnschlags) oder beleuchten ausgewählte Aspekte innerhalb einer Indikation (z.B. MS und Kognition). Zusätzlich stehen Hilfsmittel, Tests, Scores und Tabellen für Diagnose und Differentialdiagnose für den täglichen Bedarf in der Praxis bereit. Alle Informationen stehen auch zur Weiterverwendung (z. B. in Präsentationen) zur Verfügung. Das Konzept von evidentia beruht auf Neutralität und wissenschaftlicher Genauigkeit: Die Inhalte werden von Fachärzten und Opinion Leaders aus den jeweiligen Indikationsgebieten in einem mehrstufigen Prozess entwickelt und überprüft sowie regelmässig aktualisiert. Sponsoren unterstützen das Portal, haben jedoch keinen Einfluss auf den Inhalt.

evidentia startet mit dem Fachgebiet Neurologie. Das Portal wird nach und nach um weitere Indikations- und Fachgebiete erweitert, Ende des Jahres wird die französische Version aufgeschaltet. 